University of Nebraska - Lincoln DigitalCommons@University of Nebraska - Lincoln

1994

\title{
Recognition, Investigation, and Control of Communicable-Disease Outbreaks in Child Day- Care Settings
}

Jeffrey P. Davis

bureau of Public Health, Wisconsin Division of Health

William R. MacKenzie

bureau of Public Health, Wisconsin Division of Health

David G. Addiss

Centers for Disease Control and Prevention, Atlanta

Follow this and additional works at: http://digitalcommons.unl.edu/publichealthresources

Davis, Jeffrey P.; MacKenzie, William R.; and Addiss, David G., "Recognition, Investigation, and Control of Communicable-Disease Outbreaks in Child Day-Care Settings" (1994). Public Health Resources. 474.

http://digitalcommons.unl.edu/publichealthresources/474

This Article is brought to you for free and open access by the Public Health Resources at DigitalCommons@University of Nebraska - Lincoln. It has been accepted for inclusion in Public Health Resources by an authorized administrator of DigitalCommons@University of Nebraska - Lincoln. 


\title{
Recognition, Investigation, and Control of Communicable-Disease Outbreaks in Child Day-Care Settings
}

\author{
Jeffrey P. Davis, MD* $\ddagger$; William R. Mac Kenzie, MD*§; and David G. Addiss, MD, MPHI
}

\begin{abstract}
As increasing numbers of young children attend day-care centers in the US, the elevated risk of acquiring infectious diseases in this setting has emerged as an important public health issue. ${ }^{1}$ Outbreaks of infectious diseases occur frequently within the daycare setting, ${ }^{2}$ and enteric and respiratory pathogens may be readily transmitted to household members and others in the community. ${ }^{1.2}$ The economic burden of these outbreaks is considerable; for example, parents of children in day care miss an average of 1 to 4 weeks of work each year to care for their sick children. ${ }^{1}$

Investigations of communicable-disease outbreaks in day-care centers have provided a wealth of information useful in developing and implementing infection-control policies and guidelines. While documented experiences with outbreaks in day-care settings have been relatively recent, they have rapidly expanded our understanding of reservoirs of infectious agents, routes of transmission, clinical characteristics of illness, risk factors for infection, the effectiveness of interventions, and recognition of pathogens previously not reportable or thought to be unimportant. Outbreak investigations in day-care centers reported in the literature have focused primarily on the etiologic agents listed in the Table. The purpose of this paper is to provide a brief overview of methodologic issues pertinent to such investigations.
\end{abstract}

\section{CHALLENGES AND CAVEATS OF OUTBREAK INVESTIGATIONS}

Investigation and control of outbreaks in child day-care settings can be challenging and complex. Many individuals and agencies representing a variety of concerns and responsibilities are involved, including children, parents, care givers, day-care administrators, public health and regulatory agencies, and researchers. Parents, in particular, are concerned both about the health of their children and about the potential economic loss if they must miss work because their child is unable to attend day care. Second, the media may focus intense local publicity on these outbreaks, resulting in increased pressure on investigators and the need for enhanced communication. Third, day-care providers may not be receptive to participating in outbreak investigations, which can be

From the "Bureau of Public Health, Wisconsin Division of Health, Madison, WI; $\ddagger$ Departments of Pediatrics and Preventive Medicine, University of Wisconsin, Madison, WI; and §̧Division of Field Epidemiology, Epidemiology Program Office, and IDivision of Parasitic Diseases, National Center for Infectious Diseases, Centers for Disease Control and Prevention, Atlanta, GA. labor intensive, disruptive to routine day-care activities, and conducted by investigators unfamiliar to the providers. Finally, public health resources are frequently constrained and other sources of funds for these investigations are rarely available.

Outbreak investigation and control is facilitated when public health departments have established specific day-care policies and protocols that address disease prevention and control during outbreaks; when care givers and parents are knowledgeable about the public health role in outbreak intervention; when medical advice is well-coordinated; and when a single, knowledgeable spokesperson can represent the investigating team in working with the media or communicating with other persons who inquire about the outbreak.

\section{RECOGNITION OF OUTBREAKS: THE ROLE OF SURVEILLANCE}

The recognition of outbreaks or unusual health events in daycare settings is a function of disease surveillance. ${ }^{3,4}$ The sophistication and effectiveness of surveillance activities vary with the size and resources of the day-care center and the extent to which linkages have been formed with local, regional, and state public health agencies. The rapidity, efficiency, and frequency of communication among day-care officials, parents, physicians, and

TABLE. Infectious Syndromes and Etiologic Agents in Outbreak Investigations in Child Day Care

\begin{tabular}{cl}
\hline Infectious Syndrome & \multicolumn{1}{c}{ Etiologic Agent } \\
\hline Meningitis & Haemophilus influenzae, type B \\
& Neisseria meningitidis \\
& Shigella \\
Diarrhea & Escherichia coli 0157 H7 \\
& Rotavirus \\
& Adenovirus \\
& Astrovirus \\
& Giardia lamblia \\
& Cryptosporidium \\
& Hepatitis A virus \\
& Parvovirus B19 \\
\hline
\end{tabular}


public health agencies also bears greatly on the timeliness and effectiveness of surveillance.

Within the day-care setting, the most effective surveillance systems are thorough and appropriate, yet simple and based on common sense. ${ }^{3}$ Basic surveillance activities should include a daily health screening by a qualified person of each child and day-care provider upon arrival at the site. The screening should include a brief verbal review of the health status of the individual with particular emphasis on signs and symptoms of communicable diseases. Pertinent screening data should be entered into a centralized record or log book that can be used to determine if there is an unusual occurrence of illness and which also can provide valuable historic data. A designated person in the center should review the log daily.

Recognition of potential communicable disease problems is greatly enhanced when parents regularly share information about their children's health with staff members. Day-care centers should establish criteria for excluding ill children or workers from a center to reduce secondary disease transmission within the facility. Day-care providers should be aware of the need to communicate with the public health agency, and public health agencies should be involved in establishing criteria for both exclusion and communication. For example, several cases of diarrhea in the same classroom, or a single case of serious illness (eg, meningococcal disease or hemolytic uremic syndrome) should stimulate communication with the public health agency.

The established mechanisms for reporting to public health agencies offer an opportunity for surveillance of diseases in child day care. For example, on the standard Acute and Communicable Disease Report form for reportable infections in Wisconsin, information is collected on whether a person is infected with an enteric disease, is a day-care attendee, is a day-care worker, or is a member of a household in which someone attends or works in a day-care center. Timely review of this information by the local public health agency can facilitate more rapid public health recognition of enteric diseases in day-care settings.

Surveillance can be either active, enhanced or heightened, or passive. Active surveillance is intensive, regular, and prearranged contact with the day-care provider by the public health agency or other investigator. In enhanced or heightened surveillance, reporting of disease is stimulated by a specific notice but is not as intensive as active surveillance. Passive surveillance means the provider notifies the public health agency. In a study of diarrheal illness among day-care center attendees, Bartlett et al found that the initiation of active or heightened surveillance activities had the effect of decreasing the incidence of diarrhea through activities related to increased awareness. ${ }^{4}$

\section{PROTOCOL-DRIVEN OUTBREAK INVESTIGATIONS}

The collection of surveillance data and the public health response when an outbreak occurs are often based on investigation procedures and protocols. Investigators and public health agencies may prepare standing protocols in the event that a specific type of outbreak or health event occurs in a predetermined center or setting. The protocol is carefully designed to include background information, hypotheses, methods, interventions, analyses, and evaluation components. Approval of an institutional review board or human subjects committee is sought, the appropriate clearances and consents are obtained from center directors and boards and licensing and public health agencies, and provisions are made for informed consent. Research-oriented protocols involving outbreak-related events may be multicentered or community wide, ${ }^{4-6}$ and may involve day-care providers, universities, health-care providers, and public health agencies. In these types of studies, surveillance for outbreaks and the provision of prevention and control activities by nonpublic health agencies is often sanctioned. ${ }^{5,6}$

The piloting and pretesting of final survey instruments is helpful, particularly when resource expenditure is likely to be substantial. The use of standard case definitions, when applicable, facilitates comparability of data. A compendium of case definitions for epidemiologic purposes has been developed jointly by the Centers for Disease Control and Prevention (CDC) and the Council of State and Territorial Epidemiologists. ${ }^{7}$

Because of constrained laboratory and testing resources, it is critical to discuss specific needs and the anticipated magnitude of testing with laboratory personnel. When feasible and sanctioned, appropriate specimens might be collected and stored and then remain frozen for long intervals. This strategy was adopted by Lew et al when they used newly available molecular biologic assays to test stool specimens collected 5 years, previously to describe the relative importance of astroviruses and adenoviruses as etiologic agents of diarrhea among day-care attendees. ${ }^{8}$ Thus, access to stored specimens can provide critical diagnostic or historic data.

Pre-established protocols often allow for the evaluation of the epidemiologic features of asymptomatic infection and the cost effectiveness of disease control strategies. For example, Bartlett et al found that despite the higher cost of strict interventions, neither treatment or exclusion of day-care attendees asymptomatically infected with Giardia lamblia had an effect on the prevalence of $G$. lamblia after 6 months of follow-up. ${ }^{9}$

\section{OUTBREAK INVESTIGATIONS WITH NO PRE-ESTABLISHED PROTOCOL}

Outbreak investigations with standard public health responses are more typical in day-care settings. The principle goal is controlling the outbreak, which generally requires application of standard epidemiologic methods. However, to maximize the opportunity to collect new or important information, it is necessary for state and local health officials to have a working knowledge of recent developments and current research questions regarding a variety of communicable diseases and health events in day-care settings. Even for outbreak investigations in which the principle objective is disease control rather than research, it is ultimately time saving and valuable to develop and pretest standard investigation forms that facilitate objective responses, minimize bias, maximize comparability of data, and can be expanded and customized to include specific circumstances of the event under investigation.

One practical way to facilitate investigations in child-care settings is to create standardized forms that allow for collection of information common to all outbreaks. For communicable diseaserelated investigations, items can include demographic information on attendees, care givers, and family members; personal hygienic practices of care givers and attendees; and facility-specific information such as environmental sanitation, food handling procedures, ill-child exclusion practices; diapering and toileting procedures; the ratio of children to care givers, size and type of age groups; physical space; and other characteristics of the facility. In addition, it is useful to include a standard list of signs and symptoms and provide ample space for laboratory data and other important information. Benefits of such forms include ready availability and ease of customizing and expanding them for specific investigations.

For analytic purposes, Epi Info software is well-suited for use with personal computers during day-care-related outbreak investigations. ${ }^{10}$ Epi Info facilitates basic analysis and report generation. Given the many variable factors in day-care settings, multivariate analyses are often needed to test associations; data are readily exported from Epi Info to other statistical packages (eg, SAS) capable of such analyses.

\section{OUTBREAK INTERVENTION AND EVALUATION}

The successful conclusion of any outbreak investigation depends on testing the efficacy of the interventions and control measures. Elements of evaluation include follow-up testing at predetermined intervals and periodic visits and assessment by representatives of the local public health agency or other agencies to ensure that recommendations are followed and deficiencies are corrected. A report by Steketee et al demonstrates the importance of follow-up and reassessment of recommendations and interventions; the authors described the recurrence of giardiasis outbreaks in one day-care center despite extensive efforts to identify and treat persons with $G$. lamblia infections, high cure rates among treated cases, and improvement in hygienic practices. ${ }^{11}$ Compliance with regimens by parents, day-care attendees, and day-care providers are often dependent upon the perceived consequences of disease and the level of inconvenience imposed by the regimen. For example, compliance with a short course of antibiotic prophylaxis is high among persons exposed to a person with meningococcal meningitis; by comparison, compliance with a 7- to 10-day regimen for a mild Giardia infection is likely to be considerably 
lower. Determining the level of compliance is, therefore, essential to the evaluation of any public health intervention.

Investigation of multiple outbreaks attributable to the same agent in these settings has led to increased knowledge through applied epidemiologic principles. ${ }^{9,12,13}$ For example, Tauxe et al investigated outbreaks of shigellosis in two neighboring day-care centers, applied different interventions, and compared outcomes. ${ }^{12}$ Outbreaks were controlled in both centers, although the need for alternate care was 100-fold less among children attending the center in which convalescent children taking antibiotics were cared for in isolation than among children who attended the center that temporarily closed. ${ }^{12}$

\section{CONCLUSION}

The importance of disease surveillance and outbreak-control activities in child day-care settings has been detailed as part of the American Public Health Association/American Academy of Pediatrics guidelines for out-of-home day-care programs. ${ }^{14}$ Aggressive assessment of outbreaks will continue to provide critical information needed to prevent and control diseases and other adverse health events in day-care facilities.

\section{REFERENCES}

1. Thacker SP, Addiss DG, Goodman RA, Holloway BR, Spencer HC Infectious diseases and injuries in child day-care: opportunities for healthier children. JAMA. 1992;268:1720-1726

2. Klein JO. Infectious diseases and day care. Rev Infect Dis. 1986;8:521-526

3. Davis JP, Pfeiffer JA. Surveillance of communicable diseases in child day care setting. Rev Infect Dis. 1986;8:613-617

4. Bartlett AV, Jarvis BA, Ross V. Diarrheal illness among infants and toddlers in day care centers: effects of active surveillance and staff training without subsequent monitoring. Am / Epidemiol. 1988;127: 808-817

5. Pickering LK, Evans DG, DuPont HL, Vollet JJ, Evans DJ. Diarrhea caused by Shigella, rotavirus, and Giardia in day-care centers: a prospective study. J Pediatr. 1981;99:51-56

6. Van R, Wun CC, O'Ryan ML, Matson DO, Jackson L, Pickering LK. Outbreaks of human enteric adenovirus types 40 and 41 in Houston day care centers. J Pediatr. 1992;120:516-521

7. Centers for Disease Control. Case definitions for public health surveillance. MMWR. 1990;39(No. RR-13):1-43

8. Lew JF, Moe CL, Monroe SS, et al. Astrovirus and adenovirus associated with diarrhea in children in day care settings. I Infect Dis. 1991;164: 673-678

9. Bartlett AV, Englender SJ, Jarvis BA, Ludwig L, Carlson JF, Topping JP. Controlled trial of Giardia lamblia: Control strategies in day care centers. Am J Public Health. 1991;81:1001-1006

10. Dean AD, Dean JA, Burton JH, Dicker RC. Epi Info, Version 5: A word processing, database, and statistics program for epidemiology on microcomputers. Atlanta: Centers for Disease Control; 1990

11. Steketee RW, Reid S, Cheng T, Stoebig JS, Harrington RG, Davis JP. Recurrent outbreaks of giardiasis in a child day care center. Am J Public Health. 1989;79:485-490

12. Tauxe RV, Johnson KE, Boase JC, Helgerson SD, Blake PA. Control of day care shigellosis: a trial of convalescent day care in isolation. Am J Public Health. 1986;76:627-630

13. Tacket CO, Cohen ML. Shigellosis in day care centers: use of plasmid analysis to assess control measures. Pediatr Infect Dis J. 1983;2:127-130

14. American Public Health Association/American Academy of Pediatrics. Caring for our children. National health and safety performance standards: Guidelines for out-of-home child care programs. Washington and Elk Grove Village IL: APHA/AAP; 1992 